\title{
Perception of pregnant women in Ceilandia on the choice of the delivery route and the nurse's role
}

\begin{abstract}
Objective: to analyze the perception of the pregnant women about the choice of the way of delivery and the nurse's role in the prenatal guidelines.

Methodology: a qualitative study carried out in Ceilândia/DF between October and December 2013, with nine pregnant women. Semi-structured interviews were carried out and analyzed according to Bardin.

Results: they were grouped into three categories: reasons related to the choice of delivery method; knowledge of pregnant women about the birth process; and the statistics of play in the match. There was unanimous preference for vaginal delivery based on faster recovery compared to cesarean section, previous positive experience of vaginal delivery, among other factors. There was distorted knowledge about the delivery routes and poor performance of the nurse in the orientation on the birth tract. The pregnant women pointed out that there was no dialogue on the issues related to childbirth in the consultations and when they questioned the issue some professionals were unprepared to respond.
\end{abstract}

Conclusion: this study is expected to promote a change in the prenatal care model for women's empowerment and conscious choices about the way of delivery. It is believed that the professional qualification of the nurses would be one of the solutions for the increase in the quality of the assistance.

Keywords: obstetric delivery, prenatal care, nursing, personal autonomy
Volume 3 Issue 5 - 2017

\author{
Carla Gomez Rabello,' Kamilla Lorena \\ Gonçalves de Sousa,' Juliana Machado \\ Schardosim, ${ }^{2}$ Laiane Medeiros Ribeiro, ${ }^{2}$ \\ Casandra Genoveva Martins Rosales Ponce \\ de Leon, ${ }^{2}$ Alecssandra de Fátima Silva \\ Viduedo ${ }^{2}$ \\ 'Hospital Maternidade Brasília, Universidade de Brasília, Brazil \\ ${ }^{2}$ Faculdade de Ceilândia, Universidade de Brasília, Brazil
}

Correspondence: Juliana Machado Schardosim, Faculdade de Ceilândia, Universidade de Brasília, Brazil,Tel +555I 9925 50191, Email jumachadoju@hotmail.com

Received: June 29, 2017| Published: September 06, 2017

\section{Introduction}

The choice of the birth route may occur prior to gestation or until the time of delivery. Many factors go through this decision making process, which can trigger symptoms such as anxiety not only in the pregnant woman, but also in her social circle. ${ }^{1}$ At the beginning of pregnancy, this choice is usually marked by socioeconomic factors, previous childbirth experiences, the sociocultural context of the woman and information about the types of childbirth. During pregnancy, factors such as clinical obstetric complications, medical or family suggestion, and new information acquired by the pregnant woman may influence and/or modify their choice. At the time of delivery, the care received and the evolution of labor are the factors that most influence the final decision on the preference of the way of delivery. ${ }^{1}$

The increase in cesarean rates is a worldwide phenomenon, but recent studies have pointed to cesarean rates in Brazil as "abusive", "alarming" and "worrisome", well beyond the 10-15\% rate recommended by WHO an epidemic of cesarean sections for some authors. The issue is cited as a public health problem due to the repercussions for mothers and babies, such as increased rates of maternal and perinatal morbidity and mortality and impacts on breastfeeding. $^{2}$ The official Brazilian statistics of the Live Birth Information System (SINASC) points out that in 2013, only $43 \%$ of the babies were born vaginally in Brazil3. Official data from the National Supplementary Health Agency (ANS) report that in the Supplementary Health System, the average percentage of C-sections was $84.5 \%$ in the same period, with some health plan operators reporting $100 \% 4$. Due to the high rates of cesarean delivery in private health services, ANS has been developing the Adequate Childbirth
Project which, in the first phase, has already reduced cesarean rates and newborn hospitalizations in Neonatal Intensive Care Units. In addition, in June of that year (2017), WHO launched the "Waiting, Waiting" campaign in Brazil, which aims to encourage couples to wait for the onset of labor, even if they want a cesarean section. ${ }^{3}$

Looking at birth statistics in Brazil, some questions emerge: What is the reason why women are choosing both cesarean sections? Is it really that they are choosing their way? Are pregnant women and their babies so "sick" that they cannot be born/born naturally? We will try to answer some of these questions throughout the article. ${ }^{4}$

Regarding the choice of delivery method, it is recommended that the woman receive information and clarification during prenatal care so that she can make a conscious choice. In this sense, the prenatal orientation has high educational potential, since the pregnant woman becomes aware of alternative care in situations of labor without changes and, if complications arise. ${ }^{5}$ It is expected that at the end of gestation, the attending professional will evaluate with the pregnant woman the option of delivery that will best be indicated, so that an unnecessary cesarean is not performed, nor is a normal delivery assisted in risky conditions. The autonomy of the woman in the choice of the way of delivery should be maintained whenever possible, supported by her knowledge.

Professionals who work in prenatal care should provide information and clarify the pregnant woman's doubts about the types of delivery, leaving her choice free of delivery. To depreciate the patient's will or impose a will other than hers may be considered contrary to professional ethics. ${ }^{6}$ In view of the above, the objective of the study was to analyze the perception of pregnant women about the choice of the way of delivery and the nurse's role during prenatal care in the 
direction of the pregnant woman on the birth tract. The development of the study is justified due to the Brazilian statistics presented on each way of delivery.

\section{Methodology}

This is a descriptive study with a qualitative approach, carried out in a Health Center of the Administrative Region of Ceilândia, located in the Federal District. The study population was composed of pregnant women who were in the third trimester of gestation. The final sample included nine women. The selection of the sample was by convenience according to the inclusion and exclusion criteria adopted. The inclusion criteria were: age equal to or greater than 18 years and being in the third trimester of gestation. The third trimester was chosen as inclusion criterion because the authors understood that the decision on the way of delivery would have already been taken. Pregnant women 40 years of age or older were excluded because they understood that this age group tends to increase the occurrence of gestational complications that may justify cesarean section, twin pregnancy and pregnant women with a medical indication for surgical delivery.

Data collection took place through an interview with an average duration of 15 minutes guided by a semi-structured script with open and closed questions from October to December 2013. By presenting open questions the interview script was adapted to each patient. In the case of more direct pregnant women in their answers the researcher added new questions in the search for a deepening in the data researched. The closed questions corresponded to sociodemographic data and obstetric history. Data on obstetric history were searched on prenatal cards at the time of the interview.

The interviews were conducted by one of the researchers, faceto-face, in an office at the Health Center shortly after the prenatal consultation conducted by the nurse at the Unit. Only the participant and the researcher were present at the time of the interview, and occasionally some companion of the patient. The time of each participant in answering the questions was respected. Ensuring the use of the full speeches was used audio recording with subsequent transcription of the dialogue for data analysis, with permission of the women interviewed.

The data saturation criterion was used to determine the interruption of data collection. As a method of data analysis, the content analysis proposed by Bardin was used in three stages: pre-analysis; exploration of the material and treatment of results/inference/interpretation. ${ }^{7}$ Ethical aspects were respected according to Resolution 466/2012 of the National Health Council. ${ }^{8}$ The project was approved on October 7, 2013 by the Research Ethics Committee of the Health Science Teaching and Research Foundation (FEPECS) under opinion number 417.240 and CAAE no.20045413.2.0000.0030. The inclusion of the pregnant women in the study occurred by signing a Term of Free and Informed Consent. As a way of respecting anonymity women are identified throughout the text by the letter $\mathrm{G}$ corresponding to the word pregnant and numbered from 1 to 9 according to the order of the interviews.

\section{Results}

The analysis of the socio-demographic data showed that the age of the participants ranged from 19 to 37 years, the majority was single, the predominant schooling in the sample was the complete high school, the most frequent family income range was up to 1 minimum wage ( $\mathrm{R} \$ 678.00)$ and the majority were employed. The analysis of the obstetric history showed a predominant profile of Multiparous women, few of them with a history of abortion. Only one woman reported previous cesarean surgery. Three pregnant women reported intercurrences during the current gestation: bleeding, dengue and hypertension/diabetes.

Through content analysis, three categories emerged that respond to the objectives of the study.

i. Reasons related to the choice of the birth route.

ii. Knowledge of pregnant women about the birth pathways.

iii. The performance of the nurse in prenatal care.

\section{First category: reasons related to the choice of delivery method}

This category describes the reasons that influenced the choices of pregnant women regarding the way of delivery. Preference was given to vaginal delivery in all interviews, as described below.

i. I think better normal because in my family has had a very complicated cesarean case, so I'm very scared (G2).

ii. The advantage of normal delivery is the speed of recovery (G4).

iii. Oh, I already went through the first normal birth experience and going through another one was not going to be a problem (G7).

iv. The recovery is much better, so normal for me is better (G8)

It is observed in the reports that the speed in the recovery of the woman after normal delivery was an important reason in the choice by the vaginal delivery. Other factors mentioned were the fear of cesarean due to complications in family members and previous experience of successful vaginal delivery. It is noted, therefore, that personal and family experiences influence the decision-making process of the pregnant woman.

\section{Second category: knowledge of pregnant women about birth}

This category describes the knowledge of pregnant women about the birth process. The experience of the prenatal care should provide information exchange and clarification regarding pregnancy so that there is a conscious choice of the way of delivery and so that the pregnant woman feels safer when deciding her way of giving birth and giving birth properly.

Observing the reports, it is noted the lack of clarification regarding the ways of delivery, as well as lack of important information to reduce the tension of the pregnant women during the process of decision making about the way of delivery. Some reports illustrate the category:

i. Caesarean delivery has to be depending on others for everything until you recover (G5)

ii. You have to wait a long time [for normal birth]. In the noncesarean section, you program (G2)

iii. Sometimes you are afraid to expect normal birth and some problem with your baby. 
iv. The cesarean you know is born a little before [premature], but you have the security that will be born well, that will not lack oxygen (G3)

v. The best for her [the baby] I think it would be the cesarean because it does not have complications like the way it is [normal] delivery, to pull, these things ...(G8)

vi. What I do not like is what people talk about and comment on that anesthesia they give (G9)

Through the statements of the participants, incorrect information is identified, such as the impression of total control of the procedure in the cesarean section and exemption of risks for the newborn. In addition, it is not known when one of the pregnant women claims to prefer a preterm birth by cesarean section than to wait for labor and fetal hypoxia occurs. The speeches of the sample studied show that there is a lack of basic knowledge about the physiology of labor, risks, benefits and indications of each way of delivery, the importance of the labor process for the mother and the newborn, and risks associated with prematurity.

The reports confirm the need for a more active stance by health professionals (physician and nurse) in order to clarify myths and truths about the types of delivery, as well as to provide information on the risks of iatrogenic prematurity related to elective cesarean section and the importance of the passage of the fetus through the birth canal for a physiological adaptation of the baby to extra uterine life. Prenatal care is an important time to increase the knowledge of pregnant women in order to reduce cesarean rates, improve the quality of care during childbirth, encourage vaginal delivery and raise awareness about the importance of humanization in health services.

\section{Third category: the nurse's pride in prenatal care}

This category describes the perception of pregnant women about the nurse's performance during prenatal care in the clarification of the birth pathways and help in the decision-making of the pregnant woman on the choice of the birth route.

It can be noticed, by the speeches, a little acting attitude of the nurse regarding the provision of information about the birth routes and/or decision-making process of the pregnant women on the way of delivery. Some participants confessed that they never felt the need to talk to the nurse about the topic, but another commented that in a previous pregnancy the issue was addressed by the nurse, but in the current pregnancy, no. The disappointment of some pregnant women about the nurse's weak acting posture becomes visible in some of the following sections:

i. She just said that normal was better than cesarean because of the situation. But he did not say anything too much (G7)

ii. We never talked about it, so there's no telling you what I do not know (G8)

iii. They never asked me (the preference) so I never said either (G5)

iv. In other pregnancies she talked about childbirth, but a long time ago, many years (G4)

v. In the consultations she did not speak, she even asks how she is. In fact you go more for what other people talk about, not even for what she said. For now I did not feel the need to talk about childbirth with the nurse (G3) vi. The nurse never talked about my choice of delivery. I talked to my doctor who is not from the post ... (G1)

\section{Discussion}

The gestation period may represent a pleasurable time when planned, or times of anguish when unplanned. However, most of them are accompanied by fears and anxieties about an unknown future. ${ }^{9}$ The issue of childbirth has been gaining momentum in recent years. It has been observed in Brazil for decades the increasing rate of cesarean section, reaching in a worrisome magnitudes, but in the last 3 to 4 years the advances of the movement for the humanization of childbirth and birth have culminated in the stabilization of these rates and it is believed that in the near future we can celebrate the reduction of caesarean sections, for the first time in more than a decade.

Sometimes the information that Brazilian pregnant women prefer cesarean section is disclosed, but some studies report that these is a false impression and rectify this information, claiming that at the beginning of gestation the preference is for vaginal delivery and during pregnancy the option changes. The reasons that permeate the choice by elective cesarean section are numerous, often, it seems more a professional culture than a real desire of the pregnant women, being that in other situations this type of delivery is chosen by cultural aspects of the pregnant woman and her family. ${ }^{10}$

In the first category of this study "Reasons related to the choice of the way of delivery", the preference for the vaginal delivery was unanimous, however, a study shows that the women interviewed consider the choice of the delivery route a medical competence, based on the health status of the woman and baby at the time of childbirth. ${ }^{11}$ This scenario demonstrates the submission and passivity of the population served at the study site.

The choice for cesarean section is generally associated with women of high social class, white color, higher school level and consult more often during prenatal. ${ }^{12-14}$ Regardless of these factors, the desire for cesarean section, in the great majority, resembles a culture that the use of technologies in the obstetric process represents a better quality of delivery care. ${ }^{15}$ This profile of women is totally contrary to the sample studied, and consequently the opinions regarding the way of delivery as well. However, the women in this study have suggested the possibility of a cesarean birth because they feel safer for the baby, according to a study published in 2016, women are encouraged by the health system to perform a cesarean delivery because it is safer.

It is common for women who have undergone vaginal delivery to describe greater contentment, to present a more positive positioning of the delivery and to form a better bond with the baby. ${ }^{16}$ In this sample, one of the women reported having had good prior experience with normal delivery, which is why I would like to repeat the experiment.

The cesarean section profoundly impacts the relationship and the mother's feeling for the child. In a cesarean section, there is usually a mother-baby withdrawal due to the limitations imposed by the surgery, so the care demanded by the baby cannot always be performed by the mother in the first hours or days of life, breastfeeding is delayed and may influence the desire of the woman to breastfeed..$^{16}$ It was observed in this study that although women prefer normal delivery over the cesarean section that, according to them, causes a long recovery and all the consequences that arise from this lack of mobility, knowledge about the way of delivery contradicts their expectations, because most when it comes to the way of delivery consider cesarean delivery as a 
quick fix and saves them from the anxiety of going through labor and the speed with which they can safely have their baby. Often the media transmits distorted information about childbirth, creating myths about some risky situations that would indicate caesarean sections. The lay population ends up taking this information as true contributing to a social construction of a culture where cesarean delivery is a risk-free form of delivery. In this sense, it is up to the nurse during the prenatal period to exercise active listening, to know the prior knowledge of assisted pregnant women, as well as to identify gestational risks and to promote an exchange of information to understand the gestation process, providing the solution of doubts and fears about their delivery, as well as deconstruction of myths. ${ }^{17}$

However, the performance of the nurse of the unit studied in the sense of clarification about the birth pathways was a negative aspect evidenced in the discourse of the pregnant women interviewed. It was identified a little acting nurse's posture and the absence of a space of nurse-patient dialogue on the pathways of childbirth. Without an environment conducive to this openness, pregnant women do not feel free to bring their demands to the consultation, which, in a way, makes prenatal care inefficient. What is the use of pregnant women having access to consultations and examinations as recommended by the State and lacking basic knowledge about the gestational and labor process?

The approach to the issue of childbirth does not occur in an ideal way during prenatal care at the health unit studied. It was observed in the pregnant women lack of knowledge, probably due to the lack of dialogue on the subject in the consultations, a factor that impairs the conscious decision of the pregnant woman about the best way of delivery for herself and her baby. Given this scenario, the autonomy in the choice, as well as the protagonism of these pregnant women at the moment of the delivery are compromised, which can cause difficulties during the gestational cycle (gestation, delivery and puerperium), as insecurity and tension and, thus, the work can become traumatic for the woman-child and her family.

The choice of the appropriate delivery route for each pregnant woman generates a clinical discussion where the vast majority of these women do not participate, being only informed of the medical decision at the end. It does not take into account their acceptance of the behaviors to be followed, that is, women often do not participate in decisions related to their own body. From what can be seen, freedom of choice does not happen in practice and is denied with the justification of being an exclusive decision of the doctors, so that it is free of the risks that the childbirth can generate. ${ }^{18}$

Emphasis is given to the importance of a prenatal care in a holistic manner and an informed and active prenatal nurse, to deal with all subjects of interest during pregnancy, contributing to a safe choice on the part of the pregnant woman and her birth route. Dialogues and educational actions (individual or group) about the theme can contribute to a more pleasurable birth, in order to recover the protagonist role of the woman in the process of childbirth.

In $100 \%$ of the cases studied, the women reported that they did not receive any information on parturition in the current pregnancy, nor did they report on participation in orientation groups. The woman should have the right to talk with the health professional who attends her prenatal care and to participate in the choice of the path of delivery based on scientific knowledge. It is necessary to provide pregnant women with sufficient information about the advantages and disadvantages of existing birth routes so that they are able to make the best choice in a conscious way. ${ }^{19}$

Despite anxiety to deal with waiting to give birth, it is necessary to signal the baby (going into labor) to avoid maternal complications or neonatal complications. Based on this, the pregnant women should be informed about the relevance in waiting to trigger labor even though the cesarean section is their choice. In the speeches of the sample, fear was shown when waiting for vaginal delivery due to the occurrence of complications. Informed of the baby's real needs to go through labor and through the vaginal canal, it is believed that pregnant women will face this moment more peacefully, being aware that they are doing the best for their health and the health of their child.

Educational actions, developed mainly during the prenatal period, should favor this woman with comfort and security so that she can face her moment of childbirth. A woman, who undergoes an uncomplicated pregnancy and who has attended prenatal care, must be aware of the changes that have occurred at the time and be prepared for childbirth. ${ }^{9}$ Prenatal care should therefore be a time when the pregnant woman is preparing to experience a quiet, positive delivery. Lack of knowledge or misleading information about labor are factors that contribute negatively to the labor process and cause tension in pregnant women. ${ }^{9}$

The educational actions are not exclusive to the nurse, and should be performed by all health professionals who participate in prenatal care. Since 1984, with the implementation of the PAISM (Program of Integral Assistance to Women's Health), educational actions were already being taken to empower women in the proper knowledge of their bodies. This educational component was present in all subsequent Health Programs and aims to promote the participation of active in the decision-making process about the way of delivery and all the care related to the pregnancy-puerperal cycle. ${ }^{19}$

In 2011, the Stork Network was created, the current program of maternal and child health, whose objective is to improve access and quality of care from family planning, gestation, childbirth, puerperium, newborn and child up to two years of life in the public health network. Since its implementation, actions related to the humanization of prenatal care, childbirth and the puerperium have been encouraged, which strengthens the Non-Governmental Organizations that for decades have developed actions in this field, such as the Network for the Humanization of Childbirth and Birth. In addition, the Brazilian government has been striving and promoting specialization courses in Obstetric Nursing as a bet for the qualification of care provided to mothers and babies. ${ }^{20}$

The Stork Network Program should be structured in a suitable way for the health care of mothers and babies of a given territory, in a broad and satisfactory manner, guided by the principles of humanization of childbirth and birth: Organization of health services as a network of health care; shelter of the pregnant woman and the baby with risk classification at all levels of care; linkage of the pregnant woman to the maternity: pregnant woman does not peregrine, guaranteeing bed in the reference hospital at the moment of the childbirth; and routine examinations at an opportune time. ${ }^{17}$

As found in the literature, it is understood that the issue of childbirth is an essential issue to be addressed with pregnant women in prenatal consultations. The lack of dialogue and / or educational actions on the issue of childbirth is clearly identified in the reports of this sample. It is also pointed out in the reports of the pregnant women 
that some do not understand the subject as an important subject to be approached in the consultations, this fact may be related to the lack of knowledge evidenced or to the impression that they have of absence of choice on the way of delivery in the Unified Health System. It is observed that the issue of childbirth is a subject widely discussed outside hospitals and health centers, being much commented on in the family environment, friends' wheels and work environment, however we know that they are with trained professionals that pregnant women will receive correct information and will deconstruct or re-signify the myths imposed by culture. ${ }^{21}$

It should be noted that in the unit studied, the approach of the topic of delivery in the consultations was not observed, but other important orientations and procedures were performed by the nurse and she is open and available to talk with the patients about other topics. It is considered the hypothesis that the nurse considers the issue of delivery as medical responsibility, since pregnant women have consultations between doctor and nurse, but this is only a hypothesis since this was not a researched data.

\section{Conclusion}

We can conclude that the delivery method of the women studied is normal delivery, but they do not have enough information when they answered about the knowledge of the way of delivery, since they prefer normal delivery, but they identify cesarean delivery, in some aspects, more safe and convenient. In this case, the role of those who promote prenatal care is of paramount importance, since women did not have the information on parturition during prenatal care, and most worryingly, in many cases they did not consider it relevant to speak about it during the prenatal. Although this study has limitations, such as being a regional study and with a small sample, we can observe that it collaborates with the need to rethink the guidelines during the prenatal period, in order to empower women in relation to their choices in the puerperal pregnancy period.

\section{Acknowledgements}

None.

\section{Conflict of interest}

The author declares no conflict of interest.

\section{References}

1. MA Bastos Dias, RMS Madeira Domingues, AP Esteves Pereira, et al The decision of women for cesarean birth: a case study in two units of the supplementary health care system of the State of Rio de Janeiro. Cien Saude Colet. 2008;13(5):1521-1534.

2. Riscado LC, Jannotti CB, Barbosa RHS. Deciding the route of delivery in Brazil: Themes and trends in public health production. Texto e Contexto em Enfermagem. 2016;25(1):e3570014.

3. Brasil, Ministério da Saúde. Nascimentos por tipo de parto segundo região Brasília. Brazil; 2013.

4. ANS-Agência Nacional de Saúde Suplementar Brasília.
5. Amu O, Rajendran S, Bolaji II. Should doctors perform elective caesarean section on request? Maternal choice alone should not determine method of delivery. Br Med J. 1998;317(7156):463-465.

6. Barcellos LSA, Machado C. Cesariana: uma visão bioética. Rev Bioét. 2009;17(3):497-510.

7. Bardin L. Análise de conteúdo. São Paulo, Brazil; 2011.

8. Brasil. Ministério da Justiça. Conselho Nacional de Saúde. Resolução no 466. de 12 de dezembro de 2012: aprova diretrizes e normas regulamentadoras de pesquisa envolvendo seres humanos. Brasília: Diário Oficial da União; 2011. 59 p.

9. Rios CTF, Vieira NFC. Educational action in prenatal care: a reflection on nursing consultation as an opportunity for health education. Cien Saude Colet. 2007;12(2):477-486.

10. GP Barbosa, Karen Giffin, AA Tuesta, et al. Cesarean sections: who wants them and under what circumstances. Cad Saúde Púb. 2003;19(6):16111620 .

11. Liu NH, Mazzoni A, Zamberlin N, Colomar M, et al. Preferences for mode of delivery in nulliparous Argentinean women: a qualitative study. Reprod Health. 2013;10(2):2-7.

12. Gomes UA, Silva AA, Bettiol H, et al. Risk factors for the increasing caesarean section rate in Southeast Brazil: a comparison of two birth cohorts 1978-1979 and 1994. Int J of Epidemiol. 1999;28(4):687-694.

13. Maria do Carmo Leal, Silvana Granado Nogueira da Gama, Cynthia Braga da Cunhaet. Desigualdades raciais, sociodemográficas e na assistência ao pré-natal e ao parto, 1999-2001. Rev Saude Púb. 2005;39(1):100-107.

14. Paulo Fontoura Freitas, Maria de Lourdes Drachler, José Carlos de Carvalho Leite, et al. Desigualdade social nas taxas de cesariana em primíparas no Rio Grande do Sul. Rev Saude Púb. 2005;39(5):761-767.

15. Bèhague, Victora CG, Barros FC. Consumer demand for caesarean sections in Brazil: informed decision making, patient choice, or social inequality? A population based birth cohort study linking ethnographic and epidemiological methods. Br Med J. 2002;324(7343):942-945.

16. Rahnama P, Mohammadi K, Montazeri A. Salient beliefs towards vaginal delivery in pregnant women: A qualitative study from Iran. Reprod Health. 2016;13(7):2-8

17. Figueiredo B, COSTA Raquel, PACHECO Alexandra. Experiência de parto: alguns factores e consequências associadas. Análise Psicológica. 2002;20(2):203-217.

18. Brasil. Ministério da Saúde. Atenção ao pré-natal de baixo risco. Brasília: Secretaria de políticas de saúde- SPS/Ministério da saúde: Springer 2012. 320 p.

19. Tedesco R, Nelson Lourenço Maia Filho, et al. Fatores determinantes para as expectativas de primigestas acerca da via de parto. Rev Bras Ginecol Obstet. 2004;26(10):791-798.

20. Velho MB, Santos EKA, Brüggemann OM, et al. Vivência do parto normal ou cesáreo: revisão integrativa sobre a percepção de mulheres. Texto context-enferm. 2017;21(2):458-466.

21. Carneiro RG. Dilemas antropológicos de uma agenda de saúde pública: Programa rede cegonha, pessoalidade e pluralidade. Interface. 2013;17(44):49-59. 